\title{
Evaluation of a Mobile Application to Decrease Opioid Misuse and Habit-Forming Behaviors Following Prescription: Preliminary Results and Future Directions
}

\author{
Jessica Kelley Morgan ${ }^{1 *}$, Steven K. Walther ${ }^{2}$, Marian E. Lane ${ }^{1}$ \\ ${ }^{1}$ Military Behavioral Health, RTI International, Research Triangle Park, NC, USA \\ ${ }^{2}$ Continuous Precision Medicine, Research Triangle Park, NC, USA \\ Email: ^jemorgan@rti.org
}

How to cite this paper: Morgan, J. K., Walther, S. K., \& Lane, M. E. (2019). Evaluation of a Mobile Application to Decrease Opioid Misuse and Habit-Forming Behaviors Following Prescription: Preliminary Results and Future Directions. Psychology, 10, 2019-2025.

https://doi.org/10.4236/psych.2019.1015129

Received: November 13, 2019

Accepted: December 10, 2019

Published: December 13, 2019

Copyright $\odot 2019$ by author(s) and Scientific Research Publishing Inc. This work is licensed under the Creative Commons Attribution International License (CC BY 4.0).

http://creativecommons.org/licenses/by/4.0/

\begin{abstract}
Opioid misuse can, and should, be characterized as an epidemic and public health crisis. Opioid misuse, use disorders, and overdoses are costly in terms of morbidity, mortality, and humanitarian and economic costs. Managing the use of opioids in the postoperative period is an essential point of intervention in combating the opioid crisis. To address this critical issue, Continuous Precision Medicine (CPM; Research Triangle Park, NC) has developed a mobile application that tracks a patient's pain and usage of both opiates and non-steroidal anti-inflammatory (NSAID) medications to manage that pain. Participants $(N=8)$ of this feasibility study were patients undergoing outpatient surgeries in an urban area of the Southeastern United States. Seven of eight $(87.5 \%)$ patients using the mobile application began to delay the next opioid dose and took less than the recommended dosage within 24 hours post-operation, took less than half of the prescribed opioids, and consumed less than $2 / 3$ of the recommended opioids to manage their pain, thereby decreasing their plasma concentration and reducing the risk of habit-forming behaviors. Across seven of the patients, 180 oxycodone pills were prescribed, and only 39 pills (21\%) were taken, leaving 141 pills in the community. Preliminary results suggest that the CPM mobile application is feasible and acceptable for both patients and practitioners and provides traceability for the clinicians and make better-informed decisions regarding patient care.
\end{abstract}

\section{Keywords}

Opioid Misuse, Public Health, Prevention, Mobile Application, Substance 
Abuse, Pain Management

\section{Introduction}

Opioid misuse can, and should, be characterized as an epidemic and public health crisis. Opioid misuse, use disorders, and overdoses are costly in terms of morbidity, mortality, and humanitarian and economic costs. It is estimated that in 2015 alone, more than one-third of all civilian, noninstitutionalized adults living in the United States used prescription opioids and more than 11.5 million people (4.7\%) misused them (Han et al., 2017). Lifetime nonmedical prescription opioid use is estimated at $11.3 \%$ (Saha et al., 2016) and prescription opioid deaths have more than quadrupled over the past two decades (Han et al., 2017). Approximately half of the nearly 34,000 opioid-involved drug overdoses in 2015 involved prescription opioids (Rudd, Seth, David, \& Scholl, 2016); the cost of opioid overdose, abuse, and dependence in the U.S. has been estimated at $\$ 78.5$ billion annually (Florence, Zhou, Luo, \& Xu, 2016).

One risk factor related to opioid misuse and abuse is prescription following surgical procedures, both minor and major (Brummett et al., 2017). Rates of new persistent opioid use (defined as "an opioid prescription fulfillment between 90 and 180 days among those patients who filled opioid prescriptions perioperatively") have been estimated at approximately $6 \%$, with risk factors including preoperative tobacco use, alcohol and substance abuse disorders, and anxiety, among others (Brummett et al., 2017). This high incidence makes prolonged opioid use a common postsurgical complication. Additionally, several specific surgeries have been associated with increased risk of chronic opioid use (defined as "having filled 10 or more prescriptions or more than 120 days' supply of an opioid in the first year after surgery, excluding the first 90 postoperative days"), including total knee arthroplasty [TKA], total hip arthroplasty, laparoscopic cholecystectomy, open cholecystectomy, open appendectomy, cesarean delivery, and simple mastectomy (Sun, Darnall, Baker, \& Mackey, 2016). Additional risk factors for chronic opioid use among opioid-naïve patients in the postoperative period include male sex and older age (50 years or older), as well as preoperative history of depression and antidepressant use (Sun et al., 2016). Extant data also suggest that nonmedical prescription-opioid use is a risk factor for heroin use, although the majority of people who use prescription opioids for nonmedical reasons will not transition to heroin (Compton, Jones, \& Baldwin, 2016). Managing the use of opioids in the postoperative period is an essential point of intervention in combating the opioid crisis.

To address this critical issue, Continuous Precision Medicine (CPM; Research Triangle Park, NC) has developed a mobile application that tracks a patient's pain and usage of both opiates and non-steroidal anti-inflammatory (NSAID) medications to manage that pain. The application uses elements of gamification 
to encourage patients to delay medications when appropriate and to decrease dosages as able. Feasibility and acceptability studies are underway at three clinical sites. This manuscript describes the preliminary results from these studies and highlights future directions.

\section{Methods}

\subsection{Scientific Principles of Feasibility Study Designs}

This study design was guided by the objectives for assessing feasibility of social and behavioral interventions (Bowen et al., 2009; Orsmond \& Cohn, 2015). Per Bowen et al. (2009), the areas of focus of a feasibility study should include the following: acceptability (i.e., how the intended individual recipients react to the intervention); demand (e.g., patient engagement); implementation (i.e., the extent, likelihood, and manner in which an intervention can be fully implemented as planned and proposed); practicality (i.e., the extent to which an intervention can be delivered when resources, time, and/or commitment are constrained in some way); and limited-efficacy testing (i.e., testing the intervention in a limited way, including tests that may be conducted in a convenience sample, with intermediate rather than final outcomes, with shorter follow-up periods, or with limited statistical power).

\subsection{Subjects and Data Sources}

Participants were patients undergoing outpatient surgeries in an urban area of the Southeastern United States. Physicians or other medical staff offered patients the option to use the CPM mobile application in conjunction with their current standard of care. No exclusion criteria were outlined. To assess acceptability and feasibility outcomes, we leveraged two sources of data: patient assessments and the mobile application. The patient assessments assessed acceptability and were completed by patients at the end of their post-operative period. The assessment took approximately 3 minutes to complete and included questions on acceptability, usability, and perceived usefulness. The patient provided information via the mobile application on their current level of pain, usage of NSAIDs, and dosage and timing of opioid use. Subject usage data were de-identified and stored locally on the device prior to being uploaded for monitoring and analysis.

\subsection{Statistical Analysis}

All analyses were run using SAS version 9.4 software (SAS Institute Inc., Cary, NC). We used univariate analyses on data from patient assessments to examine the distribution of responses related to acceptability. To assess feasibility of the mobile application in managing pain and reducing opioid use, we examined group-level rates of opioid use and habit-forming behaviors.

\section{Preliminary Results}

Preliminary results include time to delay of next opioid dose, time to reduction 
of opioid dose, overall consumption of prescribed opioids, and plasma concentration and tolerance levels with regard to opioids. Acceptability results include ease of use and logistical feasibility for both patients and clinicians.

\subsection{Sample Characteristics}

Participants $(N=8)$ for this study were intentionally heterogenous in order to ensure feasibility and acceptability across diverse populations. The sample included both male $(n=5)$ and female $(n=3)$ patients undergoing outpatient surgeries. Surgery types included laparoscopic excision of endometriosis, femoral osteotomy, shoulder arthroscopy, lumbar discectomy, osteoplasty, knee arthroscopy, and urethrotomy. Ages ranged from 18 to 75 years old. All patients were given seven days' worth (30 pills) of opioids for post-operative pain management. Due to the limited sample size, additional demographic details are not provided to prevent identifiability.

\subsection{Preliminary Results}

\subsubsection{Decreased Opioid Use}

Preliminary data suggest that seven of eight (87.5\%) patients using the mobile application began to delay the next opioid dose and took less than the recommended dosage within 24 hours post-operation, took less than half of the prescribed opioids, and consumed less than $2 / 3$ of the recommended opioids to manage their pain, thereby decreasing their plasma concentration and reducing the risk of habit-forming behaviors.

\subsubsection{Habit-Forming Behaviors}

In the case of the patient who took the highest amount of opioid following surgery (Patient Alpha), the delaying and decreasing of the next dose mitigated overall plasma concentration (due to the half-life of the narcotics) and therefore reduced overall risk of addiction (see Figure 1). Patient Alpha's tolerance levels were also decreased across the course of post-operative treatment, thereby reducing risk of habit-forming behaviors (see Figure 2).

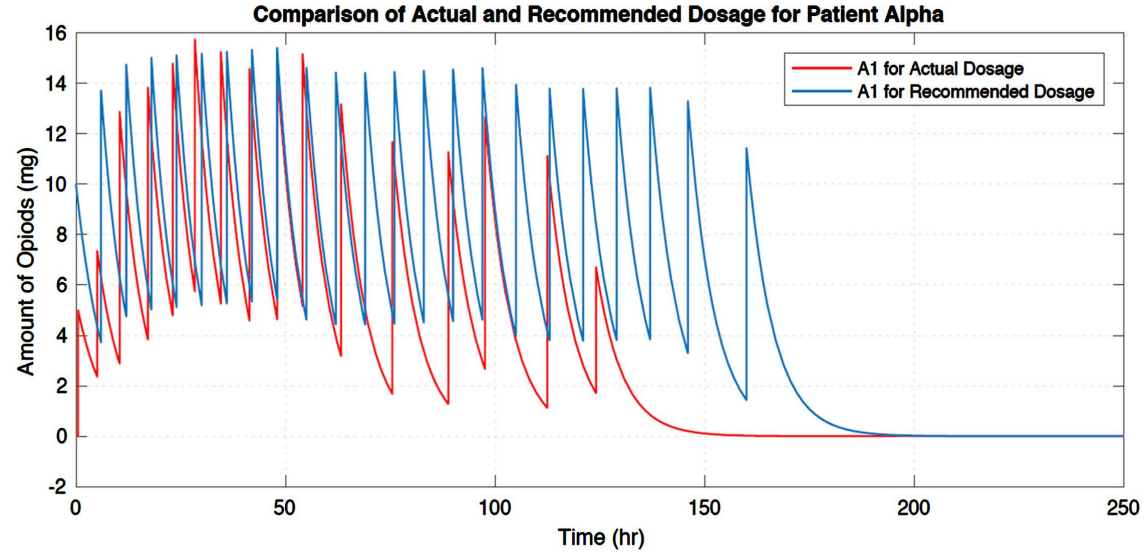

Figure 1. Comparison of actual and recommended dosage for Patient Alpha. 

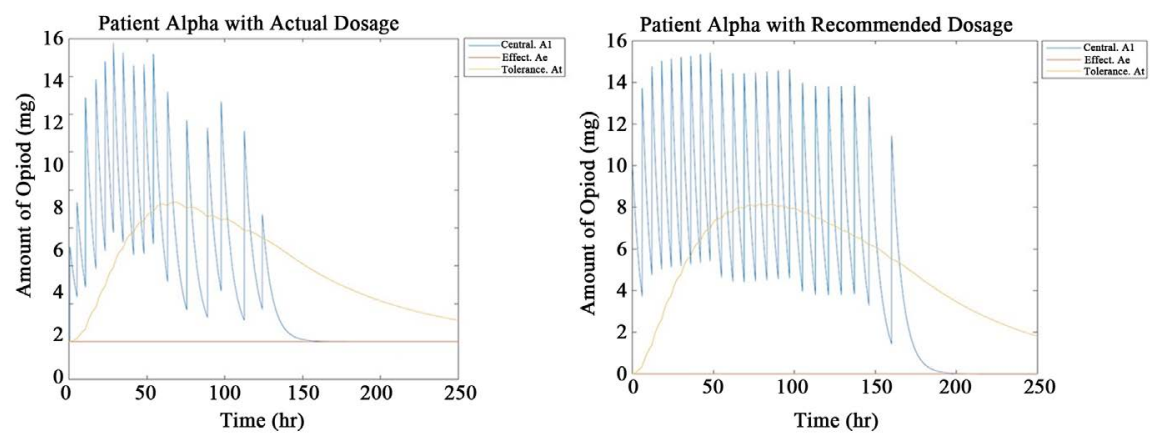

Figure 2. Comparison of tolerance levels for actual and recommended dosage for Patient Alpha.

\subsubsection{Need for Diversion of Opioids from Community}

Five out of eight (62.5\%) of the patients took 10 percent or less of the opioids they were prescribed to manage their pain, two out of eight (25\%) patients took $40 \%-50 \%$ of the opioids they were prescribed, and one patient took $86 \%$ of prescribed opioids. Across seven of the patients, 180 oxycodone pills were prescribed, and only 39 pills (21\%) were taken, leaving 141 pills in the community.

\subsubsection{Acceptability of Mobile Application Use}

All patients reported that the mobile application was easy to use, and no technical difficulties were experienced. Additionally, patients reported feeling more in control of their pain management and care plan. Clinicians reported that the inclusion of the mobile application in the post-operative care plan was not burdensome and that feedback was readily understood.

\section{Discussion}

The preliminary results reported herein suggest that the CPM mobile application is feasible and acceptable for both patients and practitioners. Our results regarding the need for diversion of opioids from the community warrant some discussion. Given that nearly $80 \%$ of the opioids prescribed were unused, it is advisable to reduce the number of pain medications being prescribed to prevent unintended consequences in patients and the community at large. Based on Morphine Milligram Equivalents (MME), the number of unused pills across only seven of the participants in this study could be fatally toxic and lead to the death of approximately 5 adults, or between 40 and 50 children (Dart, 2003; Gossel \& Bricker, 1994; Harvey, 1993), highlighting the need to right-size prescriptions.

Although not an a priori aim of this study, we also found that clinicians were surprised by the results regarding opioid consumption and the number of pills left unused by their patients as well. Several of the practitioners expressed the value of the traceability that the CPM application provides and noted that it would be helpful in their practices to understand better what happens after the prescription is written and the patient is discharged.

There are several outcomes of interest to be garnered from the CPM technology and the results of this study. Immediate outcomes include decreased usage 
of opioids among patients to manage pain and decreased risk of habit-forming behaviors. Distal outcomes include the ability to right-size prescriptions, thereby decreasing the number of prescription opioids prescribed and dispensed, as well as the ability to model pain management and risks of habit-forming behaviors to tailor pain management to reduce substance abuse and misuse.

Given the promising preliminary results from the current feasibility and acceptability studies, future directions include conducting a study that includes determining comparativeness effectiveness of CPM's mobile application vis-à-vis opioid use reduction as compared to basic monitoring via mobile application; gathering additional data on acceptability of patient engagement techniques; and modeling opioid use and pain reduction patterns.

When considering the implications of our findings, limitations of the study design should be considered. These preliminary results are based on a sample of only eight patients who were quite heterogenous in terms of age, gender, and surgical procedure. This limited sample size, for instance, does not allow for generalizability of findings across contexts. However, this study does suggest that further investigation is warranted, and that the inclusion of the CPM mobile application is feasible and likely to be acceptable for patients during the post-operative period.

\section{Conflicts of Interest}

The authors declare no conflicts of interest regarding the publication of this paper.

\section{References}

Bowen, D. J., Kreuter, M., Spring, B., Cofta-Woerpel, L., Linnan, L., Weiner, D., \& Fernandez, M. (2009). How We Design Feasibility Studies. American Journal of Preventive Medicine, 36, 452-457. https://doi.org/10.1016/j.amepre.2009.02.002

Brummett, C. M., Waljee, J. F., Goesling, J., Moser, S., Lin, P., Englesbe, M. J., \& Nallamothu, B. K. (2017). New Persistent Opioid Use after Minor and Major Surgical Procedures in US Adults. JAMA Surgery, 152, e170504-e170504.

https://doi.org/10.1001/jamasurg.2017.0504

Compton, W. M., Jones, C. M., \& Baldwin, G. T. (2016). Relationship between Nonmedical Prescription-Opioid Use and Heroin Use. New England Journal of Medicine, 374, 154-163. https://doi.org/10.1056/nejmra1508490

Dart, R. C. (Ed.). (2003). Medical Toxicology(3rd ed.). Philadelphia, PA: Lippincott Williams \& Wilkins.

Florence, C. S., Zhou, C., Luo, F., \& Xu, L. (2016). The economic Burden of Prescription Opioid Overdose, Abuse, and Dependence in the United States, 2013. Medical Care, 54, 901. https://doi.org/10.1097/mlr.0000000000000625

Gossel, T. A., \& Bricker, J. D. (1994). Principles of Clinical Toxicology (3rd ed.). New York: Raven Press.

Han, B., Compton, W. M., Blanco, C., Crane, E., Lee, J., \& Jones, C. M. (2017). Prescription Opioid Use, Misuse, and Use Disorders in U.S. Adults: 2015 National Survey on Drug Use and Health. Annals of Internal Medicine, 167, 293. 
https://doi.org/10.7326/m17-0865

Harvey, A. L. (Ed.). (1993). Natural and Synthetic Neurotoxins. London: Academic Press.

Orsmond, G. I., \& Cohn, E. S. (2015). The Distinctive Features of a Feasibility Study: Objectives and Guiding Questions. OTJR: Occupation, Participation and Health, 35, 169-177. https://doi.org/10.1177/1539449215578649

Rudd, R. A., Seth, P., David, F., \& Scholl, L. (2016). Increases in Drug and Opioid-Involved Overdose Deaths-United States, 2010-2015. MMWR. Morbidity and Mortality Weekly Report, 65, 1445-1452. https://doi.org/10.15585/mmwr.mm655051e1

Saha, T. D., Kerridge, B. T., Goldstein, R. B., Chou, S. P., Zhang, H., Jung, J., \& Grant, B. F. (2016). Nonmedical Prescription Opioid Use and DSM-5 Nonmedical Prescription Opioid Use Disorder in the United States. The Journal of Clinical Psychiatry, 77, 772-780. https://doi.org/10.4088/jcp.15m10386

Sun, E. C., Darnall, B. D., Baker, L. C., \& Mackey, S. (2016). Incidence of and Risk Factors for Chronic Opioid Use among Opioid-Naive Patients in the Postoperative Period. JAMA Internal Medicine, 176, 1286-1293.

https://doi.org/10.1001/jamainternmed.2016.3298 